\title{
Traffic Noise and Maximum-Flow Spanning Trees on Growing and Static Networks
}

\author{
Bosiljka Tadić ${ }^{1}$ and Stefan Thurner ${ }^{2}$ \\ ${ }^{1}$ Department for Theoretical Physics, Jožef Stefan Institute, \\ Box 3000, 1001-Ljubljana, Slovenia \\ ${ }^{2}$ Complex Systems Research Group, HNO, Medical University of Vienna, \\ Währinger Gürtel 18-20, A-1090 Vienna, Austria
}

\begin{abstract}
Properties of traffic noise and flow are often measured on complex networks and are used to diagnose the network's functional state and underlying structure, even though the precise structure-function interdependences are often unknown. Here we attempt to unravel some basic interdependences between structure and traffic on networks in numerically controlled traffic models. We simulate constant-density traffic on two different network topologies, which emerge from the same preferential rewiring rules but one within growth and the other under static conditions. We determine universal noise properties and the maximalflow spanning trees on these classes of network topologies. We study both low-density traffic (structure dependences) and high-density traffic, where queuing influences transport properties.
\end{abstract}

\section{Introduction}

Dependences of network topology and optimal network functioning are gaining increased attention in network research lately [1,2,3. While networks driving e.g. biological processes have adapted and optimized their structures through evolutionary mechanisms, whose details are largely unknown [4, artificial networks, on the other hand, often suffer from all kinds of dis-functional problems and weaknesses. Both theoretical and practical aspects of the structure-function interdependences are of interest.

In real networks, measurements of dynamic variables, such as flow through individual links, fluctuations of noise at individual nodes, round-trip times of information packets, high-throughput measurements etc., are often used to diagnose the network's functional state and structure. The idea is that the temporal fluctuations of the local node and link activities in an intricate way reflect the underlying network structure and potential dynamic correlations. The nature of these interdependences, however, is often hidden in the non-linearity of the dynamics and in most cases not known. In the absence of precise theoretical grounds, often largely simplified formal procedures are applied for these purposes, often severely missing crucial aspects of the studied systems. It is therefore of great importance to identify the essential elements of the structure-function interdependences in complex networks in a systematic manner. One approach is 
to use numerical models of network dynamics in which a controlled variation of both structural parameters and the dynamic rules can be done systematically and inter-correlations can be detected and quantified. Moreover, robustness can be checked against varied dynamic conditions.

Here we apply an approach of this kind to study information transport on networks within a numerical model with driving, search, and queuing which we introduced in Refs. [5, 6, 7]. We use two classes of networks which are grown from the same preferential rewiring rules and same control parameters, but with different conditions that are known as evolving and static networks. By implementing the traffic rules on these networks, we show how they perform the information transport within the same dynamical conditions of a stationary traffic flow. Within the model we measure the travel times of packets, flow at all links on the network and temporal fluctuations of all node activities. In the low-density limit of our dynamics when packet density $\rho \rightarrow 1$, representing the infinitely slow driving (or sequential packet dynamics), these measures incorporate most of the network structural effects on the transport. In the high density limit we measure waiting times of packets at individual nodes and global noise correlations, which give quantitative estimates of the queuing effects on networks. In the low-density limit we present quantitative results for the flow intensity along network links, which defines the maximum-flow spanning tree of the network, as well as noise correlations and queue distributions, when the packet density is kept high. We show how the observed properties of traffic are related to the structural characteristics of both types of networks.

\section{Structures and Traffic Rules}

\subsection{Network Structures}

We consider two types of networks grown from preferential attachment [8] and rewiring rules [9], in which we apply different constraints known as evolving and static conditions. $(i)$ In the evolving case, the network is grown by sequentially added nodes from $i=1,2, \cdots N$, with the preferential attachment and preferential rewiring rules according to the time dependent probabilities $p_{\text {in }}$ and $p_{\text {out }}$. They are applied in the subset of pre-existing nodes at each growth step $i$. The linking probabilities depend on the current number of incoming $q_{i n}$ and outgoing $q_{\text {out }}$ links at a node 9

$$
p_{\text {in }}(k, i)=\frac{\alpha+q_{\text {in }}(k, i) / M}{(1+\alpha) i} \quad p_{\text {out }}(n, i)=\frac{\alpha+q_{\text {out }}(n, i) / M}{(1+\alpha) i} .
$$

These rules lead to power-law distributions of both incoming and outgoing links [9], a large clustering coefficient and link correlations [10], a structure which is statistically very similar to the actual WWW. This is why this network is called Web graph (WG). An example of the emergent structure is shown in the left column of Fig. 1. (ii) For the static graph we apply the same rules as in Eq. (11), however, the links are added sequentially for the fixed number of nodes $i=N$. 

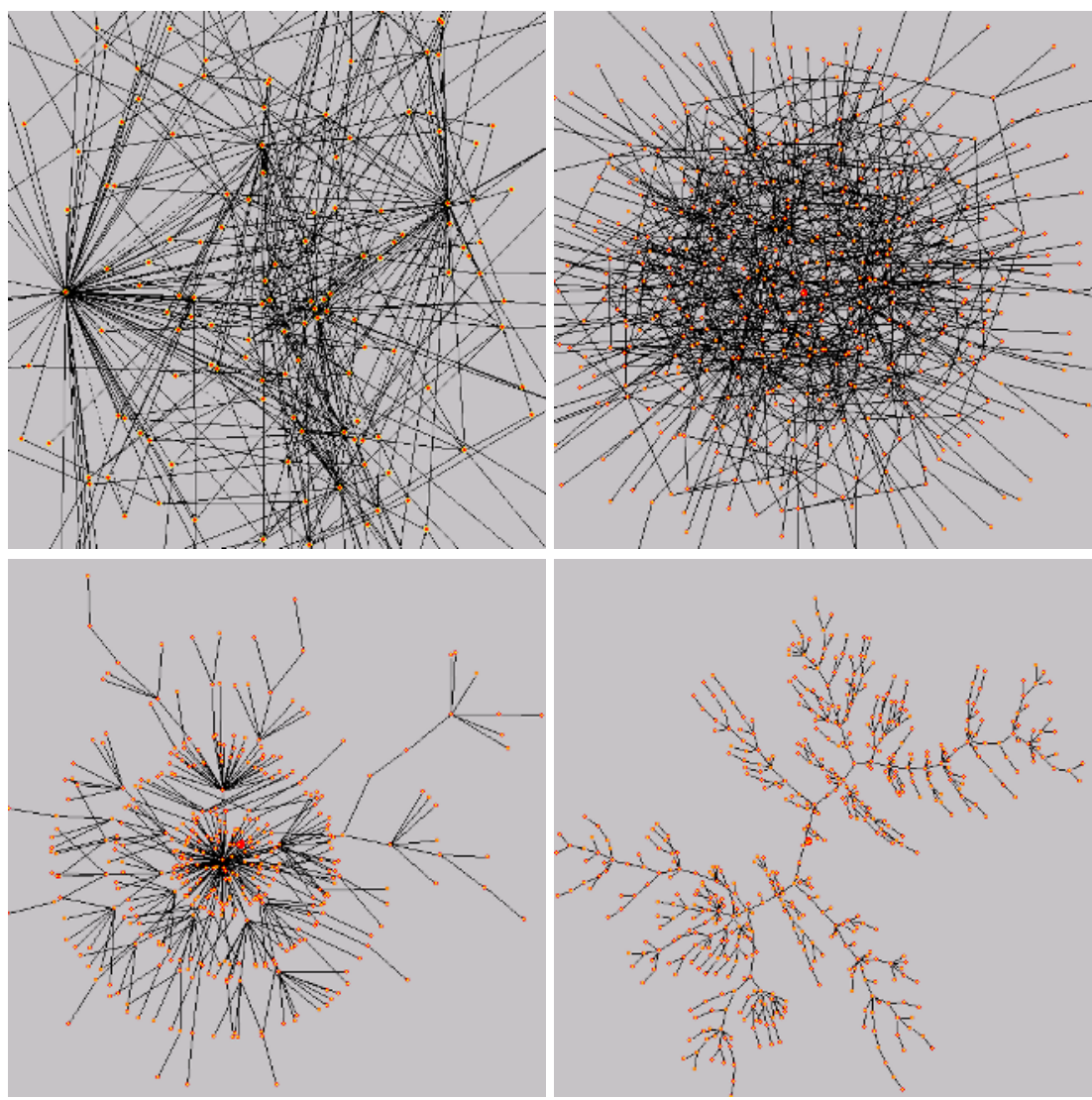

Fig. 1. Top row: Core of the cyclic scale-free Web graph (WG), left, and preferential static network (SN), right. Bottom: Maximum-flow spanning trees of the above graphs, obtained in constant-density traffic $\rho=1$, with $n n n$-search.

Multiple linking between the same pair of nodes is not allowed. The emergent structure of the static network (SN) where $L=N=1000$ links is also shown in Fig. 1 (right).

A detailed quantitative analysis of the structure reveals that both incoming and outgoing links behave statistically the same and obey a stretched-exponential distribution. In addition, the clustering in this graph is small compared to the Web graph and link correlations are entirely absent. In Fig. 2 we show the degree distributions for the two graphs.

\subsection{Implementation of Constant-Density Traffic}

The traffic of information packets on a network is implemented as a guided random walk between specified pairs of nodes on the graph [5, 17, 10] - the origin and destination (delivery address) of a packet. The created packets are navigated 


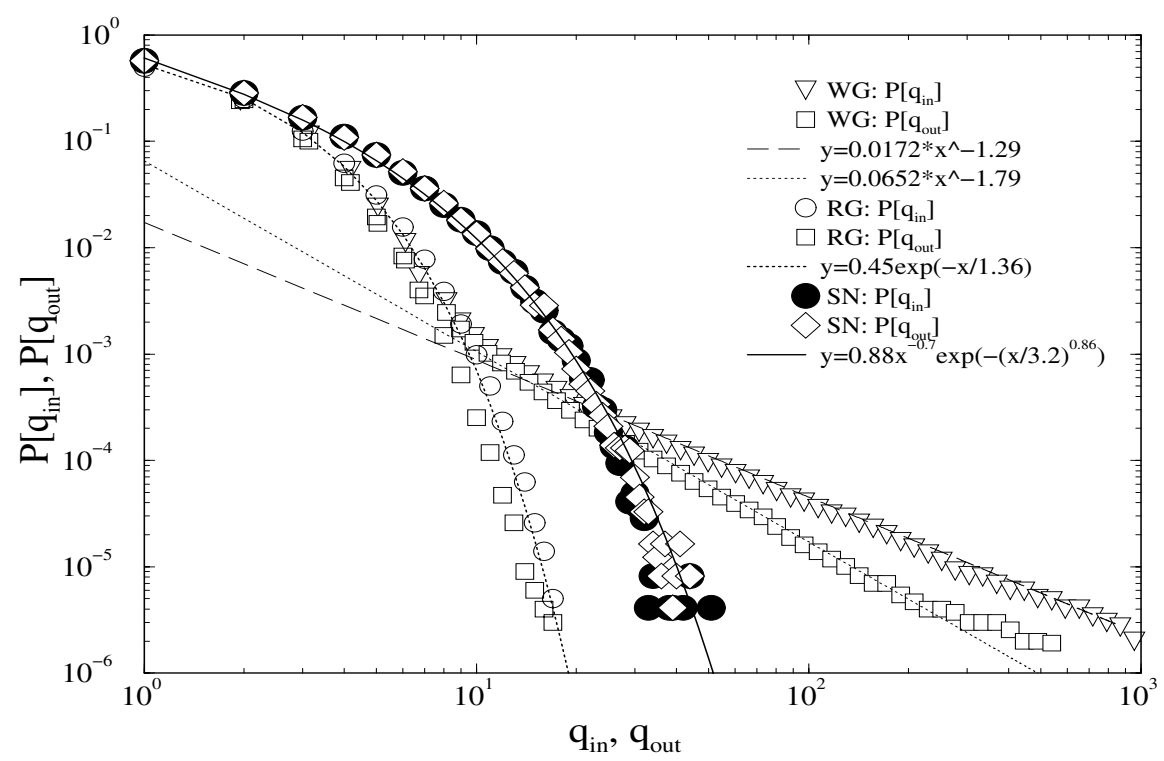

Fig. 2. Degree distribution for incoming and outgoing links in the Web graph (WG) with its power-law tails 9], the static net (SN) exhibiting broad stretched-exponentials, and the random graph (RN), for $N=L=10^{3}$ nodes and links

through the graph using the local nnn-search rule [5, 10, where two depth levels around each node (sometimes called information horizon 2) are searched for the packet destination address. The rule is supplemented by random diffusion when the search is unsuccessful. The packets are removed when they arrive at their destinations. Here we implement the traffic for a fixed number of moving packets. We start with a given number $\rho$ of packets. The arrived and removed packets are replaced in the next time step by creating the same number of new packets at randomly chosen nodes. In the limit $\rho=1$, this corresponds to the sequential random walk problem. At density $\rho>1$ packets interact by forming queues at nodes along their paths. We assume finite maximum queue lengths of $H=1000$, and a LIFO (last-in-first-out) queuing rule. The length of an actual queue at node $i$ we denote by $h_{i}(t)$. Networks are given by their adjacency matrix. When the graphs are disconnected, as it is the case with both WG and SN, we consider the transport only within their giant clusters.

\section{Low-Density Traffic: Limit $\rho=1$}

The limit of infinitely slow driving $\rho \rightarrow 1$ is easily realized: as one packet arrives at its destination, another one is created somewhere else in the network. Therefore, packet interaction is absent and the properties of traffic entirely depend on network topology. 
By running a large number of packets we record the number of walks along each link (dynamic flow) and through each node (dynamic noise) in the network. Obviously, the inequality of nodes with respect to their local network environment makes the flow and noise fluctuate throughout the network and makes it differ among different network structures. One approach toward a quantitative analysis of these fluctuations is to construct a maximum-flow spanning tree, on which each node is connected to the rest of network nodes via its maximum-flow link. Implementing a greedy algorithm, we determine the trees respective to the flow at $\rho=1$ limit on the two networks. The trees are shown in Fig. 1 (bottom).

The structure of these trees reflects both the underlying network geometry and how that geometry effects transport with given navigation rules-local $n n n$ search. In the case of the WG the tree exhibits a scale-free topology, suggesting a certain degree of compatibility of the traffic and structure. Similarly, for the SN the tree shows some inhomogeneity that resembles the one on the underlying graph.

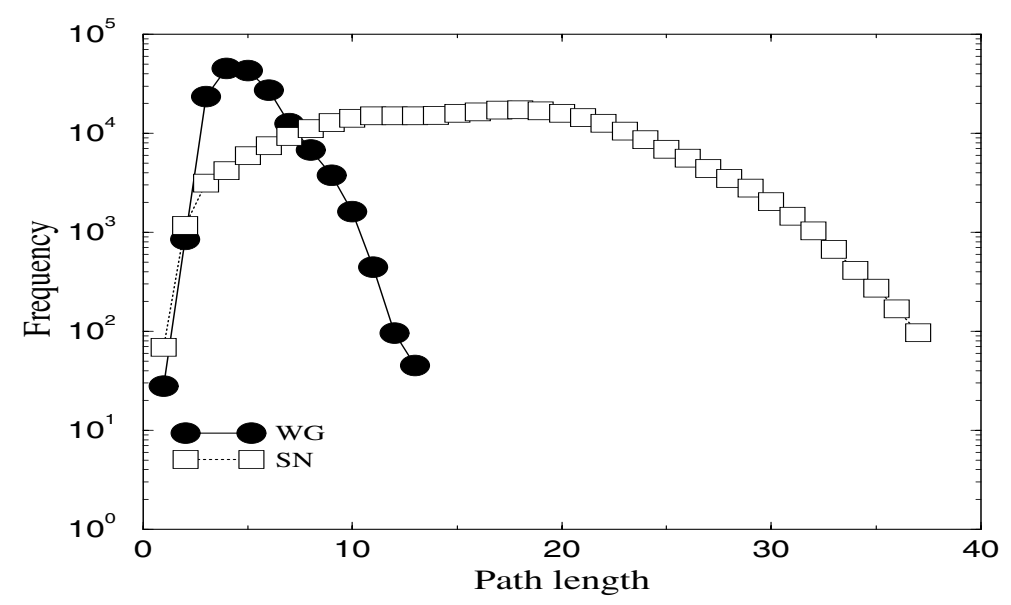

Fig. 3. Distance distributions on the maximum-flow spanning trees for the WG and the SN for $\rho=1$, and $n n n$-traffic

The max-flow spanning trees represent the union of maximum-flow paths on the underlying network structure. In Fig. 3 we show distributions of lengths of all such paths on the two trees that are shown in Fig. 1. Once again, differences in the graph topologies and thus in their maximum traffic trees manifest themselves in the statistics of the maximum-flow paths. The average distance along such paths on the WG and SN differs by a factor of about 5, the maximum distance by about a factor of 3 .

\section{Noise Properties for High-Density Traffic}

For large packet density $\rho>>1$ motion of a packet may be affected by other packets moving through the same node. Queues of packets then occur and a 
priority rule sets the order of processing (LIFO as a particular case). Apart from the traffic density, the node surrounding on the network determines the length of the queue at that node. In particular, on inhomogeneous networks, hubs appear to receive more packets compared to other nodes, due to their large connectivity. Since in the algorithm one packet is processed per time step, other packets remain in the queue to be processed later (when no new packet is received). The distribution of queue lengths is therefore reflecting the network structure in a particular way. A snapshot of queue-lengths $h$, for traffic density $\rho=100$ in the two network structures WG and SN leads to the distributions shown in Fig. 4.

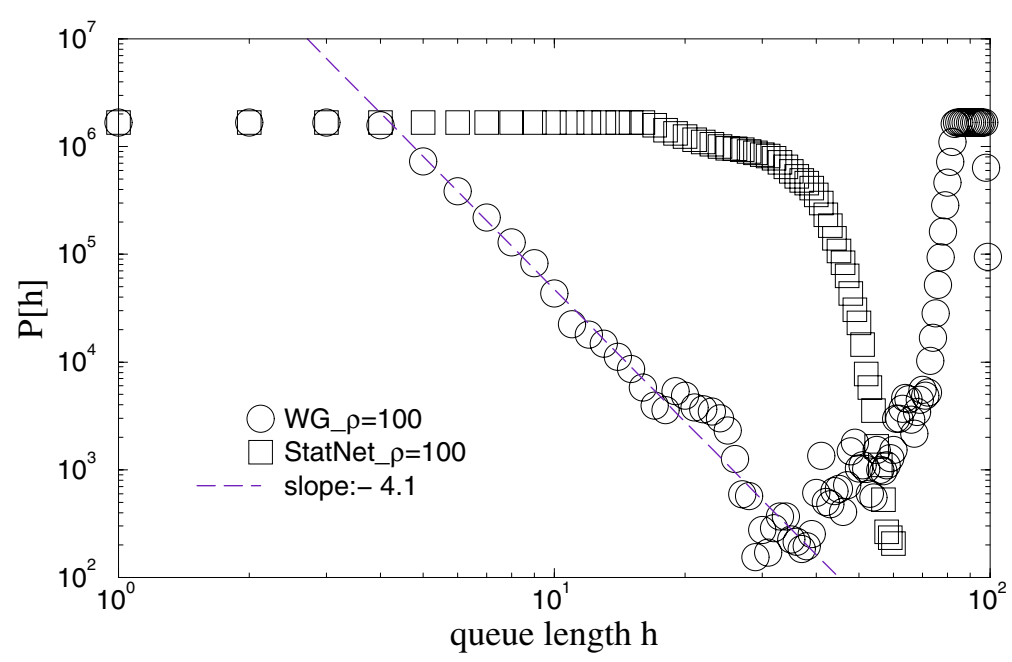

Fig. 4. Distributions of queue-lengths in the WG and the SN for a density $\rho=100$

In the homogeneous SN most of the nodes are processing a similar numbers of packets, which leads to a flat distribution of queues and a cut-off indicating that queues larger than $h=40$ are occurring rarely. On the other hand, a large queue of $h=80-90$, packets can be found on the hubs on the inhomogeneous WG with high probability. On the rest of the nodes the queues are distributed with a power-law distribution, apart from very small queues at periphery nodes. The queuing times of packets extend their travel times, thus reducing the overall traffic efficiency [7]. Note, that in the current implementation, with a constant number of moving packets, jamming can not occur as long as the traffic density $\rho<H$, where $H$ is the maximum allowed queue length. However, the travel times of packets can be very long (given by a power-law distribution) [5].

The observed queue lengths are compatible with the the temporal properties of node activity on the two networks, shown in Fig. 5. While queues at important nodes in the inhomogeneous WG are long, the number of nodes that are simultaneously active is small, fluctuating about an average value $n \approx 8$. 

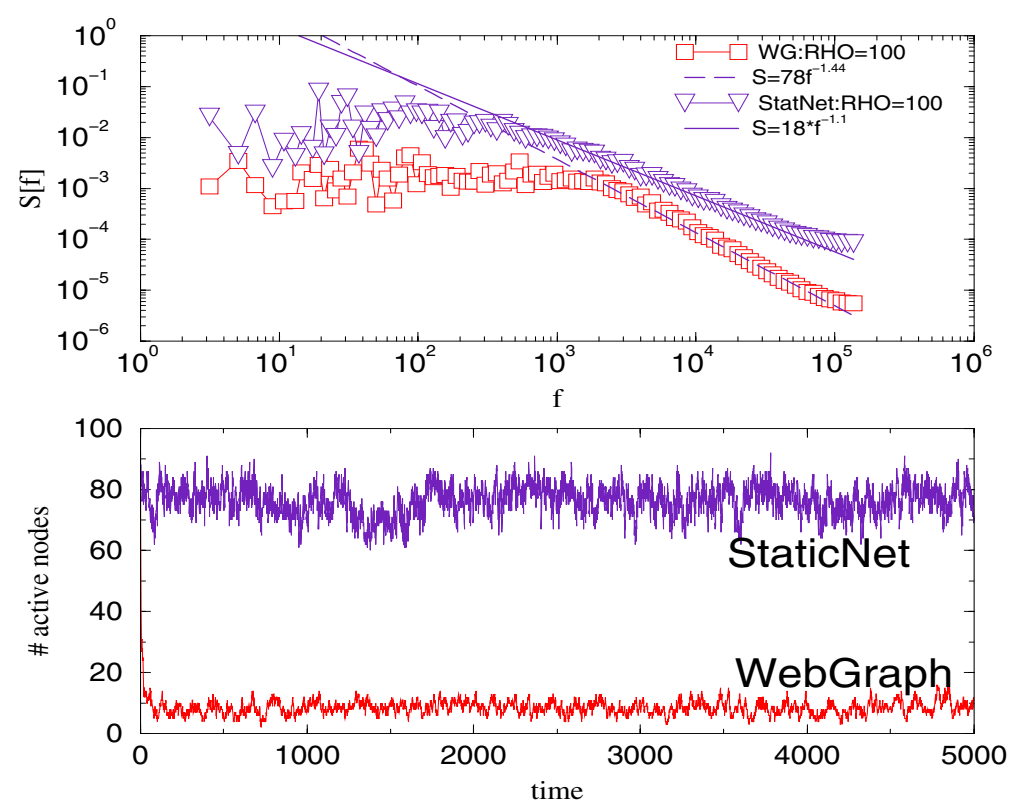

Fig. 5. Temporal fluctuations of the number of active nodes in WG and SN (lower panel) and their power spectra (top panel) for a density of $\rho=100$ packets and with nnn-search

Compared to the more homogeneous $\mathrm{SN}$ for the same traffic density, on average $n \approx 80$ nodes are processing a packet simultaneously, leading to short queues at all nodes. Further quantitative analysis of the time series reveals the differences in the packet processing of the two classes on networks. In particular, long-range correlations (anti-persistence) in the number of active nodes develops on both networks. However, in the conditions of constant packet density the fluctuations on the SN appear to be more correlated compared to the WG. The power spectrum exhibiting a power-law behavior $S(f) \sim f^{-\phi}$ for $f$ above some threshold value, are given in Fig. 5 (top panel), with $\phi=1.1$ for SN and $\phi=1.4$ for the WG. Therefore, an increased traffic density leads to stronger correlations among node activity on the more homogeneous SN. Differences are also observed in the noise fluctuation properties on these networks, which will be reported elsewhere.

\section{Conclusions}

We have implemented a constant-density information-packet traffic on two classes of network topologies: correlated, strongly clustered scale-free WG and a more homogeneous, weakly clustered uncorrelated SN. We have shown that for different driving conditions and the $n n n$-search strategy the WG tends to form large queues at its hub nodes, whereas, the more homogeneous SN distributes traffic over a large number of nodes, whose activity appears to be 
strongly correlated in time. Therefore despite of generally larger distances as illustrated e.g. with the maximum-flow spanning tree, the homogeneous network performs well at high traffic density.

Acknowledgments. BT thanks for support from the Program P1-0044 of the Ministry of high education, science and technology, Slovenia; ST for the FWF Project P17621, Austria. Partial support by the bilateral project SI-AT/01/0405 and the COST P10 action is also acknowledged.

\section{References}

1. Newman, M. E. J.: The structure and function of complex networks. SIAM Rev. 46 (2003) 167-225

2. Boccaleti, S., Latora, V., et al.: Complex Networks: Structure and Dynamics, Physics Reports 424 (2006) 175-306

3. Guimera, R., Diaz-Guilera, A., Vega-Redondo, F., Cabrales, A., Arenas, A.: Optimal network topologies for local search with congestion. Phys. Rev. Lett. 86 (2001) 3196-3199

4. Kaneko, K., On recursive production and evolvability of cells: Catalytic reaction network approach, in Geometric Structures of Phase Space in Multidimensional Chaos: A Special Volume of Advances in Chemical Physics, Part B, edited by M. Toda et al., 103 (2005)Ch.27, p. 543-598

5. Tadić, B., Thurner, S.: Information Super-Diffusion on Structured Networks. Physica A 332 (2004) 566-584; cond-mat/0307670

6. Tadić, B., Rodgers, G.J.: Packet transport on scale-free networks. Advances in Complex Systems 5 (2002) 445-456

7. Tadić, B., Thurner, T., Rodgers, G. J.: Traffic on complex networks: Towards understanding global statistical properties from microscopic density fluctuations. Phys. Rev. E 69 (2004) 036102

8. Dorogovtsev, S.N., Mendes, J.F.F.: Evolution of Networks: From Biology to the Internet and the $W W W$, Oxford University Press, 2003. Ch.27, p. 543

9. Tadić, B.: Dynamics of directed graphs: the world-wide Web. Physica A 293 (2001) 273-284

10. Tadić, B.: Modeling Traffic of Information Packets on Graphs with Complex Topology. Proceedings ICCS 2003, P. Sloot et al. Eds., Lecture Notes in Computer Science, Springer (Berlin) Part I, 2657 (2003) 136-142

11. Bollobás, B.: Modern Graph Theory. Springer (New York) 1998 